\title{
Sleeve Gastrectomy with Intra-operative Endoscopic Guidance
}

\author{
Mohamed I Hassan, Yasser El Ghamrini, Mohammed Attia Elsayed \\ Department of General Surgery, Ain Shams University, Egypt
}

Purpose: To assess the advantage of using intraoperative endoscopic guidance during sleeve gastrectomy and its efficiency to decrease various complications.

Methods: This was a retrospective study at Ain shams specialized hospital and Cure hospital. Patients who had sleeve gastrectomy with intra-operative endoscopic guidance were included in this study.

Results: From September 2017 to February 2019, 226 patients had LSG as a definitive bariatric procedure. All the procedures were done with intra-operative endoscopic guidance. Seventy nine percent were female with a mean age of $39.7 \pm 14$ years (range $23-49$ years) and a mean body mass index of $48.6 \pm 14 \mathrm{~kg} / \mathrm{m} 2$ (range $41-59 \mathrm{~kg} / \mathrm{m2}$ ). Two cases showed intraoperative leak that was managed intraoperatively by over suturing and omental patch. One case showed mild twisting at the incisura angularies that was managed intraoperatively by fixing the twisted angle to the retroperitoneum. Marked internal bleeding from the staple line was seen in two cases; which necessitated endoscopic clip insertion in one case and epinephrine injection for the other. The average length of the hospital stay was 1.8 days post operatively. The post-operative leakage was $0 \%$ and no cases had obstructive symptoms.

Conclusion: Sleeve gastrectomy surgery with endoscopic guidance is very important not only to calibrate the size of the gastric pouch instead of the bougie, but also to prevent various complications as gastric obstruction or bleeding or even fatal complications as gastric leak.

Key words: Sleeve, Gastrectomy, Endoscopy, Twist, Leak.

\section{Introduction}

Laparoscopic sleeve gastrectomy is very poplar bariatric procedure after it had been considered the first step of two staged duodenal switch operation. ${ }^{1}$

Laparoscopic sleeve gastrectomy in spite of its simplicity, it has many complications as gastric fistula in 2.5 percent of cases, bleeding in 5 percent and gastric stenosis in 0.69 to 3.5 percent of cases. ${ }^{2}$ Gastric stenosis is classified either functional or organic. ${ }^{3}$ Functional stenosis is also called gastric twist. Gastric twist is classified according to the time of diagnosis into intraoperative diagnosis, early postoperative and delayed postoperative diagnosis. ${ }^{4}$ Gastric leaks can be due to mechanical or ischemic causes. Stapler misfiring, or direct tissue injury can cause leak that usually appear within 2 days of surgery. ${ }^{5}$ It's recommended to use a bougie., also the size of the bougie correlate with the leak rate. ${ }^{6}$ Our protocol was using 36 for bougie, lately we are using endoscopic guidance during sleeve gastrectomy.

Aim: To assess the advantage of using intraoperative endoscopic guidance during sleeve gastrectomy and its efficiency to decrease various complications.

\section{Materials and methods}

This was a retrospective study at Ain shams specialized hospital and Cure hospital including 226 patients. Informed consent was obtained; the procedure and all the possible complications were explained to the patients.

Data was acquired from a retrospective review of the records of patients who underwent LSG between September 2107 and February 2019. Preoperative patient characteristics as age, sex, weight, body mass index, comorbid conditions, operative factors (operating time), and intraoperative and postoperative events, including staple line leaks, bleeding and post-operative obstructive symptoms were recorded.

\section{Surgical Technique}

We are using 4 ports technique. Division started 5 $\mathrm{cm}$ from the pylorus, once dissection of the greater omentum was completed; we started using the endoscope as guidance instead of the bougie.

We had been using a PENTAX endoscopy. The outer diameter of the scope is $10.1 \mathrm{~mm}$; equivalent to 30 for bougie. The endoscope was introduced under vision and was anchored on the first part of the 
duodenum. After complete aspiration of fluids and air of the stomach, we started firing of the stapler 1 $\mathrm{cm}$ from the edge of the scope. (Fig. 1).

We were using 5- 7 stapler cartilages (Echelon 60 endopath stapler, endoscopic linear cutter-straight, Ethicon Endo-surgery Inc.). Equal traction of the anterior and posterior wall of the stomach was ensured.

After completion of gastric resection (Fig. 2), the endoscope assessed the patency of the gastric tube, or any bleeding from the gastric pouch (Figs. 3,4). We did air bubble test using the insufflation of the scope. If any leak was detected, over sewing the leakage site was done with reinforced omental patch. We didn't over sew the suture line or fix the pouch to the retroperitoneum. All the cases had been done laparoscopically and endoscopically by the same bariatric team.

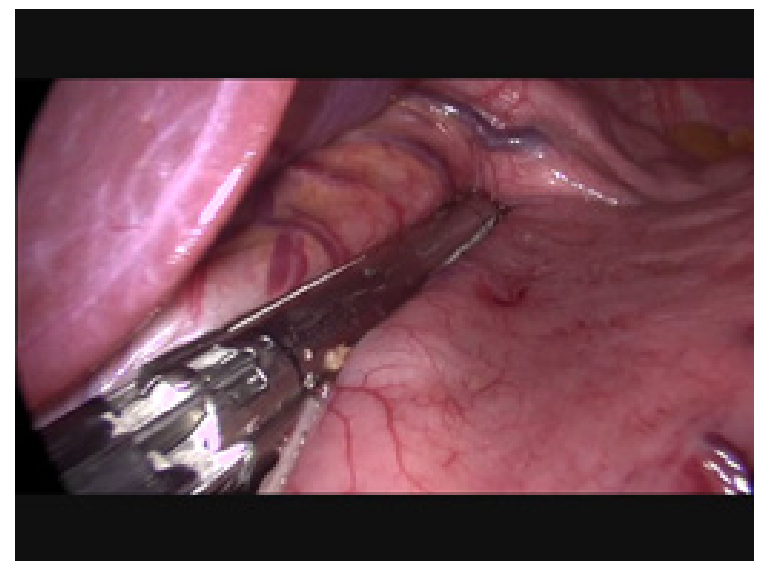

A

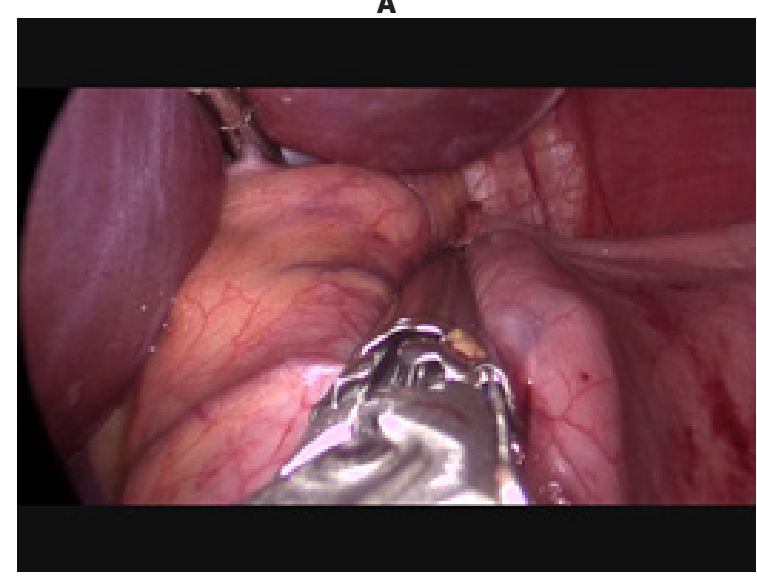

B

Fig 1a,b: Firing of the stapler $1 \mathrm{~cm}$ from the edge of the scope.

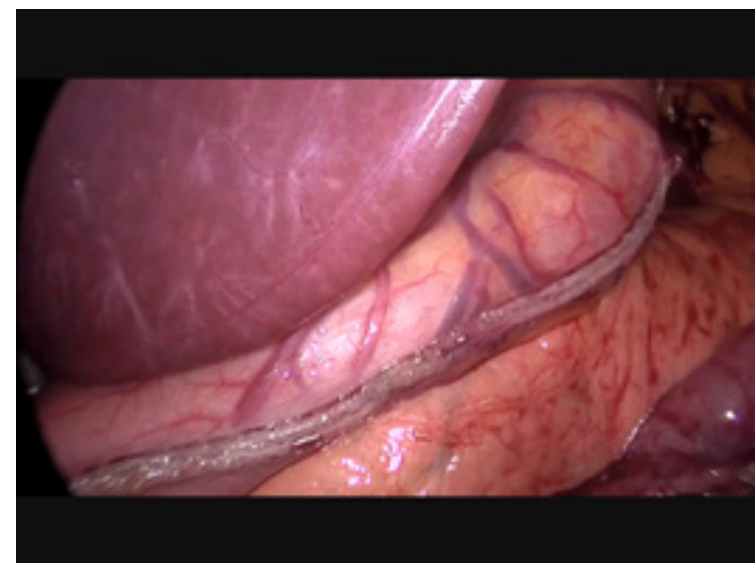

Fig 2: Completetion of gastric resection.

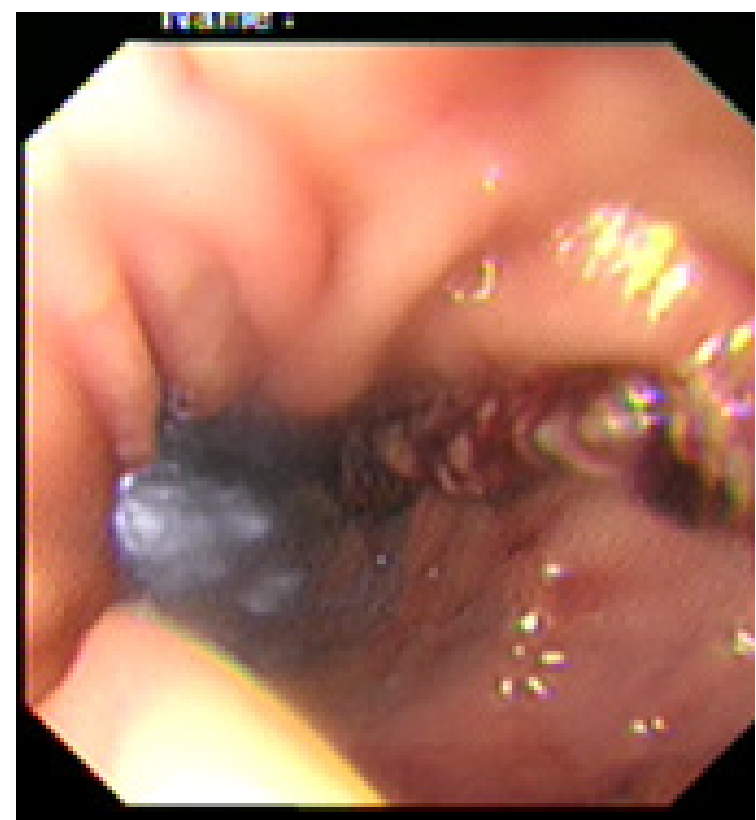

Fig 3: Endoscopic assessment of the suture line with laparoscopic illumination appear at the distal pouch.

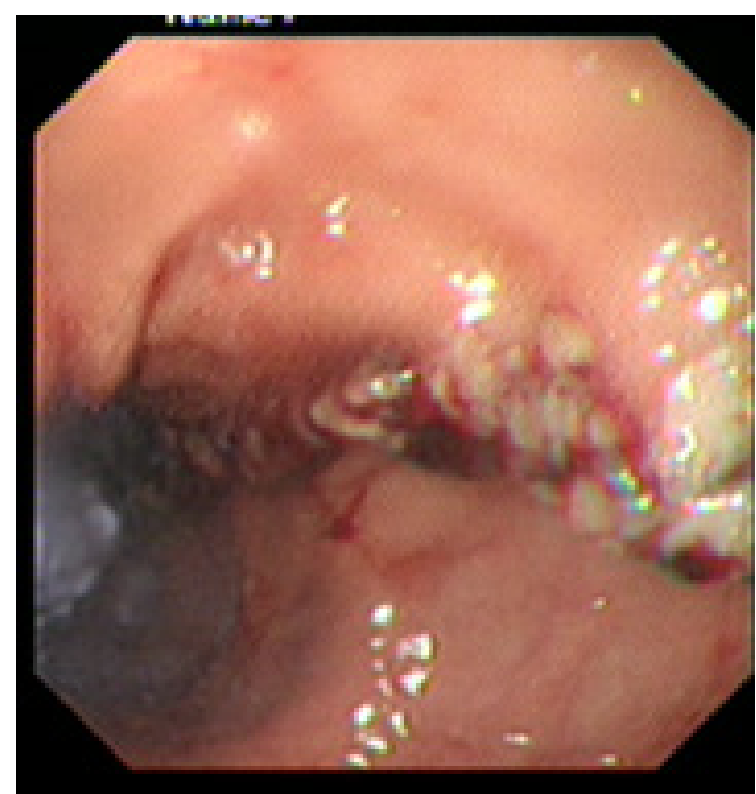

Fig: 4 Endoscopic assessment of the suture line with laparoscopic illumination appear at the distal pouch. 


\section{Results}

The study involved 226 patients who underwent LSG as a definitive bariatric procedure. Seventy nine percent were female. The mean age was $39.7 \pm 14$ years (range 23-49 years) with mean body mass index of $48.6 \pm 14 \mathrm{~kg} / \mathrm{m} 2$ (range $41-59 \mathrm{~kg} / \mathrm{m} 2$ ). 2 cases had showed intraoperative leak that was managed successfully intraoperatively by over suturing and omental patch. One case had mild twisting at the incisura angularies that was managed by fixing the twisted angle to the retroperitoneum. 2 cases had marked internal bleeding from the staple line which necessitated endoscopic clip insertion for one case and epinephrine injection for the other. The average length of the hospital stay was 1.8 days post operatively. The leakage was $0 \%$ and no cases had obstructive symptoms.

\section{Discussion}

Sleeve gastrectomy procedure with intra-operative endoscopy detected the patency of the gastric sleeve and excluded the presence of any twist or organic obstruction of the gastric pouch.

Stenosis rate of the gastric pouch in our study is $0 \%$ which is highly comparable to the published rates of stenosis after laparoscopic sleeve gastronomy under bougi guidance. The stenosis rate after LSG in the published literature ranges from $0.7 \%$ as published by Lacy et al up to $4 \%$ with Cottom et al study. ${ }^{7,8}$

Nimeiri et al reported $0 \%$ clinical stricture rate despite having $3.2 \%$ intraoperatively detected strictures. He attributed that to the routine use of intraoperative endoscopic guidance. Presence of obstruction increases the risk of leakage post operatively. ${ }^{9}$

While it is difficult to assess the presence of a stenosis at the incisura laparoscopically, this can easily be detected using intra operative endoscopy.

Laparoscopic sleeve gastrectomy with endoscopic guidance was first described by Freez et al in only 20 patients with no complications. ${ }^{10}$

Kockerling and Schug-Pass had 38 patients who had sleeve gastrectomy under endoscopic guidance; the reported postoperative complications were in 2 patients (5.2\%): A staple line bleeding and a stenosis. ${ }^{11}$

Diamantis et al described 25 patients undergoing this procedure under endoscopic guidance without morbidity and mortality. ${ }^{12}$

Although we had 2 cases with intraoperative leak, the 2 cases were managed intraoperatively. Our post-operative leak was $0 \%$ which is highly comparable to the results of Ruiz-Tovar et al. They reported $2 \%$ leakage rate. ${ }^{10}$

There are 2 cases $(0.8 \%)$ in our series had internal bleeding from the internal staple line that were managed by intra-operative endoscopy. Post-operative internal bleeding rate and blood transfusion was $0 \%$ which is highly comparable to the published literature that range from $1-6 \% .{ }^{11}$

Our results are similar to Kockerling and Schug-Pass who had 2 cases with internal bleeding that were managed by adrenaline injection. ${ }^{11}$

Intra-operative endoscopy needs an expert endoscopist as the patient lie on supine position rather than the usual left lateral position for endoscopy; also the passage of the endoscope to the duodenum is much more difficult, as the greater curvature is completely dissected and the stomach is mobile not anchored as in normal endoscopy.

The intra-operative endoscopic guidance was performed by a member of our bariatric team under vision rather than the anesthesiologists who introduce the boogie blindly, which eliminated the possibility for esophageal perforation.

The endoscopic guidance has diminished the risk for upper esophageal perforation, when compared with the blind insertion of the bougie. ${ }^{12}$

The use of an endoscope in order to calibrate the diameter of the gastric pouch allowed us to assess how close we lie to the lesser curvature, as its light facilitates the correct placement of the instrument with numerous advantages which can reduce the morbidity and mortality of patients who underwent LSG.

The results of this study had showed that the use of intraoperative endoscopy helps in detecting various complications intraoperatively and allow us to manage them rather than manage such complications postoperatively, longer hospital stay, blood transfusion and other interventions either endoscopic or operative intervention.

We believe that sleeve gastrectomy surgery with endoscopic guidance is very important not only to calibrate the size of the gastric pouch instead of the bougie but also prevent the complications as gastric obstruction or bleeding or even fatal complications as gastric leak.

\section{References}

1. Himpens J, Dobbeleir J, Peeters G: Long-term results of laparoscopic sleeve gastrectomy for obesity. Ann Surg. 2010; 252: 319-324.

2. Burgos AM, Csendes A, Braghetto I: Gastric 
Stenosis After Laparoscopic Sleeve Gastrectomy in Morbidly Obese Patients. Obes Surg. 2013; 23(9): 1481-1486.

3. Gluck B, Movitz B, Jansma S, et al: Laparoscopic sleeve gastrectomy is a safe and effective bariatric procedure for the lower BMI (35.0$43.0 \mathrm{~kg} / \mathrm{m} 2$ ) population. Obes Surg. 2011; 21(8): 1168-1171.

4. Elias S, et al: Gastric Twist after Laparoscopic Sleeve Gastrectomy, Diagnosis and Management: A Case Series and Discussion. Diabetes Obes Int J. 2017; 2(3). doij-16000159.

5. Baker RS, Foote J, Kemmeter P, Brady R, Vroegop T, Serveld M: The science of stapling and leaks. Obes Surg. 2004; 14: 1290-1298.

6. Rebibo L, Hakim S, Dhahri A, Yzet $T$, Delcenserie R, et al: Gastric Stenosis After Laparoscopic Sleeve Gastrectomy: Diagnosis and Management. Obes Surg. 2016; 26(5): 995.

7. Lacy A, Ibarzabal A, Pando E, et al: Revisional surgery after sleeve gastrectomy. Surg Laparosc Endosc Percutan Tech. 2010; 20: 351-356.

8. Cottam D, Qureshi FG, Mattar SG, et al: Laparoscopic sleeve gastrectomy as an initial weight loss procedure for high-risk patients with morbid obesity. Surg Endosc. 2006; 20: 859-863.

9. Nimeri A, Ibrahim M, Maasher A, Al Hadad: The Use of Intraoperative Endoscopy May Decrease Postoperative Stenosis in Laparoscopic Sleeve Gastrectomy. M. Obes Surg J. 2016; 26(1): 2125.

10. Frezza EE, Barton A, Herbert $H$, Wachtel MS: Laparoscopic sleeve gastrectomy with endoscopic guidance in morbid obesity. Surg Obes Relat Dis. 2008; 4: 575-579.

11. Kockerling F, Schug-Pass C: Gastroscopically controlled laparoscopic sleeve gastrectomy. Obes Facts. 2009; 2: 15-18.

12. Diamantis T, Alexandrou A, Pikoulis $E$, et al: Laparoscopic sleeve gastrectomy for morbid obesity with intraoperative endoscopic guidance. Immediate peri-operative and 1-year results after 25 patients. Obes Surg. 2010; 20: 1164-1170.

13. Lowham ES, Filipi CJ, Hinder RA, et al: Mechanisms and avoidance of esophageal perforation by anesthesia personnel during laparoscopic foregut surgery. Surg Endosc. 1996; 10: 979-982. 